\title{
Numerical Study for Laser Source Protection from Alpha Particles by Magnetic Fields in a Laser Fusion Reactor*)
}

\author{
Yoshihiro KAJIMURA, Takayoshi NORIMATSU ${ }^{1)}$ and Hideki NAKASHIMA ${ }^{2)}$ \\ Japan Aerospace Exploration Agency, 3-1-1 Yoshinodai, Sagamihara, Kanagawa 252-5210, Japan \\ ${ }^{1)}$ Institute of Laser Engineering, Osaka University, 2-6 Yamada-oka, Suita, Osaka 565-0871, Japan \\ ${ }^{2)}$ Dept. Advanced Energy Engineering Science Kyushu University, Kasuga, Fukuoka 816-8580, Japan
}

(Received 7 December 2010 / Accepted 16 March 2011)

\begin{abstract}
A possible method for protecting beam ports and laser sources from alpha particles which are produced by a nuclear fusion in the fast ignition laser fusion power plant (KOYO-Fast) is proposed. Two simple dipolar magnetic fields generated by two coils installed at the tip of the beam port and the side of the beam port are used for protecting the alpha particles coming into the inside of the beam port and colliding to the tip surface of the beam port. To calculate the behavior of alpha particles in the magnetic field, we use a 3D hybrid numerical simulation code. As a result, the intensity of $0.9 \mathrm{~T}$ of the magnetic flux density at the tip of the beam port is large enough for achieving the $10 \%$ reduction of the collision energy of the alpha particles to the tip surface of the beam port compared with the case without protection. Furthermore, the intensity of $1.35 \mathrm{~T}$ of the magnetic flux density at the center of the coil installed at the side of the beam port can guide the alpha particles coming into the inside of the beam port to the outside liquid wall perfectly.
\end{abstract}

(C) 2011 The Japan Society of Plasma Science and Nuclear Fusion Research

Keywords: KOYO-Fast, laser beam source protection, alpha particle, magnetic field, simulation

DOI: $10.1585 /$ pfr.6.2404049

\section{Introduction}

The laser fusion power plant (KOYO-F) driven with cooled-Yb:YAG, ceramic lasers was conceptually designed by Norimatsu et al. [1]. The design of this reactor is based on the fast-ignition scheme. The KOYO-F is a more realistic power generation plant with smaller power output than the previous plant KOYO [2].

One of important design issues is a design of a chamber. Damage of the first wall by irradiation of low velocity alpha-particles is the most serious problem [2]. As a protection of the first wall, $\mathrm{LiPb}$ liquid wall chamber has been proposed. On the other hand, naked laser beam ports are not protected. For protecting the beam ports from alphaparticles, one idea has been proposed such that covering the front of the beam port with the porous metal. The liquid metal oozes out from the inside and the beam ports are protected from the thermal load by keeping the surface colder to enhance condensation of $\mathrm{LiPb}$ vapors in every shot. However, under the condition that the wall temperature of beam port becomes around $873 \mathrm{~K}$, the adsorption rate on the liquid metal does not so change even if the surface temperature keeps low temperature by the cooling. So, it has been considered that this idea is not so reliable about the protection of beam ports.

In our past research [3], we proposed a method of protection from fusion-produced alpha particles by using an

author's e-mail: kajimura@gd.isas.jaxa.jp

*) This article is based on the presentation at the 20th International Toki Conference (ITC20). artificial magnetic field generated by the hoop coil. We conducted a numerical simulation for evaluating the reduction of alpha particles by changing intensity and configuration of the magnetic field. The targeted reduction level of alpha particles is less than $10 \%$ of the load without protection by the magnetic field. As a result, the intensity of $0.9 \mathrm{~T}$ of magnetic flux density at the tip of beam port is large enough for achieving the $10 \%$ reduction. On the other hand, there were some alpha particles coming into the beam port directly, and the simple dipolar magnetic field generated by a hoop coil could not protect these alpha particles. We proposed a new configuration of magnetic field generated by the eight rectangle coils [4]. By controlling the each current of eight coils, the strong verticalcomponent of the magnetic field to the direction of the beam line is generated and can perfectly prevent all alpha particles from coming into the inner beam port. However, the amount of energy colliding to the surface of beam port increased more than $10 \%$ using this configuration of magnetic field.

In the present paper, in order to guide the alpha particles coming into the beam port to the first wall by passing the alpha-particles through the hole which is drilled on the beam port surface and in order to protect the inside of the beam port and the laser source perfectly, a new method is proposed that an additional hoop coil along the laser beam port is installed. We estimate the intensity of the magnetic flux density at the center of the additional ring coil which 
can guide the alpha particles coming into the beam port to the first wall.

\section{Simulation Code and Model}

To calculate the behavior of alpha particles in the magnetic field, we use a 3D hybrid numerical simulation code which treated ions as individual particles and electrons as a fluid. This code is based on the model given by Harned [5].

A schematic calculation model is shown in Fig. 1. The simulation region is indicated as a dotted square in this figure. A predicted amount of alpha particles from fusion plasma is $7.1 \times 10^{19}$ particles/shot. The calculated flux of alpha particles near a nozzle of beam port is $6.3 \times 10^{17}$ particles $/ \mathrm{m}^{2}$. So the alpha particles produced by the fusion are emitted with the velocity of $1.4 \times 10^{6} \mathrm{~m} / \mathrm{s}$, number density of $3.5 \times 10^{18} / \mathrm{m}^{3}$ and radiation thickness of $0.25 \mathrm{~m}$ as shown in Fig. 1. The size of beam port is also shown in the right side of Fig. 1. For reducing the calculation cost, it assumes that the alpha particles flow with the constant velocity from the fusion point to the region whose distance is $2.7 \mathrm{~m}$.

The amount of alpha particles produced by the nuclear fusion near the beam port is calculated based on the fusion energy of $200 \mathrm{MJ} /$ shot in KOYO-F. The simulation is conducted under the assumption that alpha particles are emitted in all directions isotropically, although the alpha particle distribution depends on the irradiation direction of ignition beam to the fusion target or existence of guide cone with target fuel.

The coil configuration of each case is shown in Fig. 2. In Case A, one simple hoop coil is located at the tip of the beam port. The current of this coil is $I_{\mathrm{A}}=180 \mathrm{kA} \cdot$ turn and its frequency is $4 \mathrm{~Hz}$. In Case $\mathrm{B}$, as a new configuration, the additional one hoop coil is installed along the beam port as shown in the right side of Fig. 2. The current of this additional coil is changing from $I_{\mathrm{B}}=0$ to $738 \mathrm{kA} \cdot$ turn. The lower panels of Fig. 2 show the cross section of vector plot of the initial magnetic field generated by the coils in each case. This cross section is indicated at the center of the beam port ( $Y=0, X Z$ plane). The vector plot of the magnetic field in Case A shows the dipolar magnetic field and the only $z$-component of the magnetic field can be seen at the center of the coil. This magnetic cusp causes the behavior of alpha particles coming into the beam port. The same configuration of magnetic field of Case B can be seen in the right panel of Fig. 2. The additional coil can generate the strong $x$-component of magnetic field and it is expected that alpha particles can be guided to the first liquid wall if a hole is drilled on the cylindrical beam port wall in order to pass the alpha particles through this hole.

The calculation parameters are listed in Table 1. The plasma beta which is the ratio of plasma kinetic energy to the energy of the magnetic field is less than unity in every case, so the magnetic field in every case can reflect back the alpha particles generated by the fusion in the reactor. The Larmor radius (calculated by the intensity of

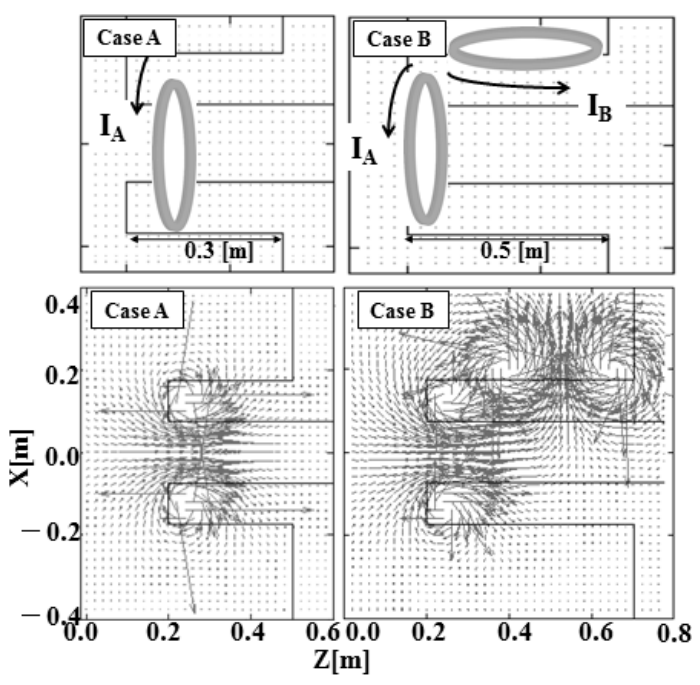

Fig. 2 Coil location and vector plot of magnetic field. Case A: Single coil (Main coil), Case B: Main coil and Additional coil.

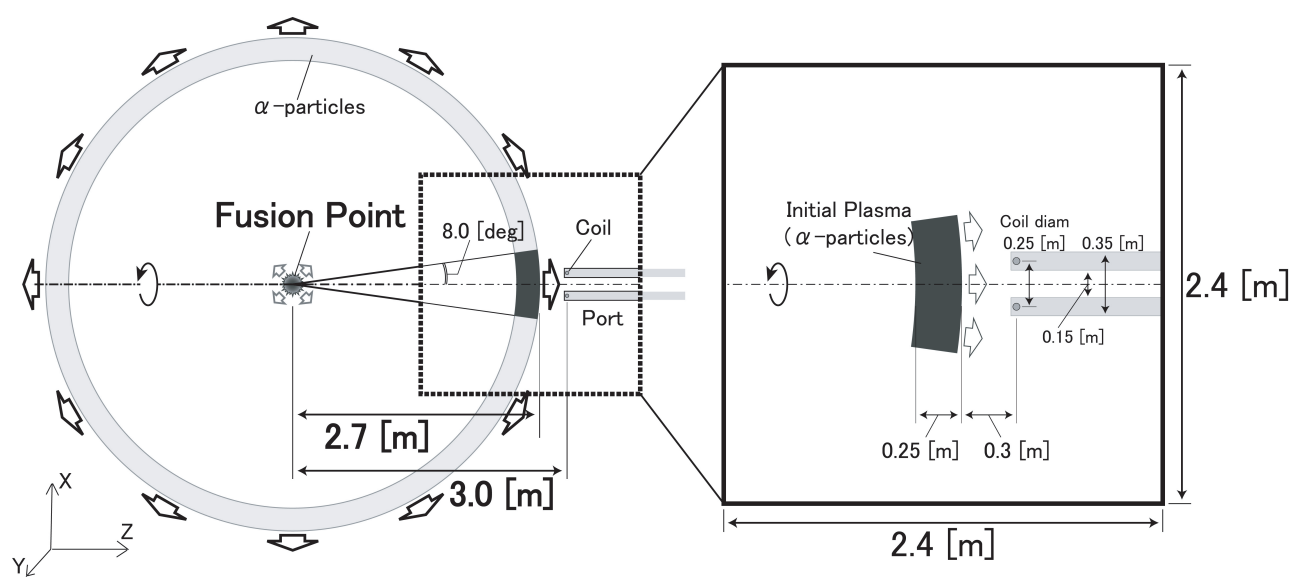

Fig. 1 Simulation Model. 
Table 1 Simulation Cases and Parameters.

\begin{tabular}{|c|c|r|r|r|r|}
\hline $\begin{array}{c}\text { Case } \\
\text { name }\end{array}$ & $\begin{array}{c}\text { Radius of } \\
\text { main, } \\
\text { Secondary } \\
\text { Coil }[\mathrm{m}]\end{array}$ & $\begin{array}{r}\text { Intensity of the } \\
\text { magnetic field } \\
\text { at the center of } \\
\text { coil (Main, } \\
\text { Second) }[\mathrm{T}]\end{array}$ & $\begin{array}{c}\text { Current of } \\
\text { each coil } \\
\left(I_{\mathrm{A}}, I_{\mathrm{B}}\right) \\
{[\mathrm{kA} \cdot \text { turn }]}\end{array}$ & $\begin{array}{c}\text { Plasma } \beta \\
\text { at the } \\
\text { center of } \\
\text { main coil }\end{array}$ & $\begin{array}{c}\text { Larmor } \\
\text { Radius [m] } \\
\text { at the } \\
\text { center of } \\
\text { main coil }\end{array}$ \\
\hline Case A & $(0.125,0.0)$ & $(0.9,-)$ & $(180,0)$ & 0.007 & 0.033 \\
\hline Case B & $(0.125,0.26)$ & $(0.9,0.9 \sim 1.8)$ & $\begin{array}{r}(180,370 \sim \\
738)\end{array}$ & 0.007 & 0.033 \\
\hline
\end{tabular}

magnetic field at the center of the coil (main coil) installed at the tip of the beam port) of alpha particle in each case is comparable or greater than the size of radius of inner beam port $r=0.075 \mathrm{~m}$, so the ion kinetic effect should be considered. The calculation step size is equal to 0.001 of the ion cyclotron frequency. The total calculation time is $0.7 \mu$ s which corresponds to around $34 \omega_{\mathrm{ci}} t$. The grid size is $0.02 \mathrm{~m}$ which corresponds to $0.16 c / \omega_{\mathrm{pi}}$ in all cases and total number of simulation particles is $2.0 \times 10^{5}$, which corresponds to put 15 particles in each cell of the initial plasma region.

\section{Simulation Results}

Figure 3 shows the distribution of alpha particles at $t$ $=0.53 \mu \mathrm{s}$ and the vector plot of the magnetic field in the same plane shown in Fig. 2. The magnetic field acts like a spring to push back the fusion plasma. This effect can be seen in all cases. The black dots shown in Fig. 3 indicate the alpha particles existed in the range from $y=-0.075 \mathrm{~m}$ to $y=0.075 \mathrm{~m}$ whose distance is equal to the inner radius of the beam port. In Case A, the tip surface of the beam port can be protected from alpha particles by the magnetic field. However, a part amount of the alpha particles passed to the $+Z$ region across the center of the main coil. This is because the magnetic field generated by the main coil has a magnetic cusp at the center of the main coil. In Case A, a lot of alpha particles coming into the beam port impinge to the inside surface of the cylindrical beam port since the direction of the magnetic field vector in the beam port is the direction to the wall of beam port. On the other hand, other alpha particles coming into the beam port do not impinge to the inside surface of the beam port and they flow toward the laser source as shown the Case A in Fig. 3. In Case B, alpha particles coming into the beam port can be guide to the outside of the beam port since the strong $x$-component of magnetic field which is generated by the additional coil. These alpha particles guided to the outside by this additional magnetic field will be treated by the liquid first wall. It is expected that alpha particles can be guided to the first liquid wall if a hole is drilled on the cylindrical beam port wall in order to pass the alpha particles through the hole as shown in Fig. 4.

Figure 5 shows the percentage of the energy of alpha particles in each region normalized by the each energy in the case without magnetic field. The definition of the

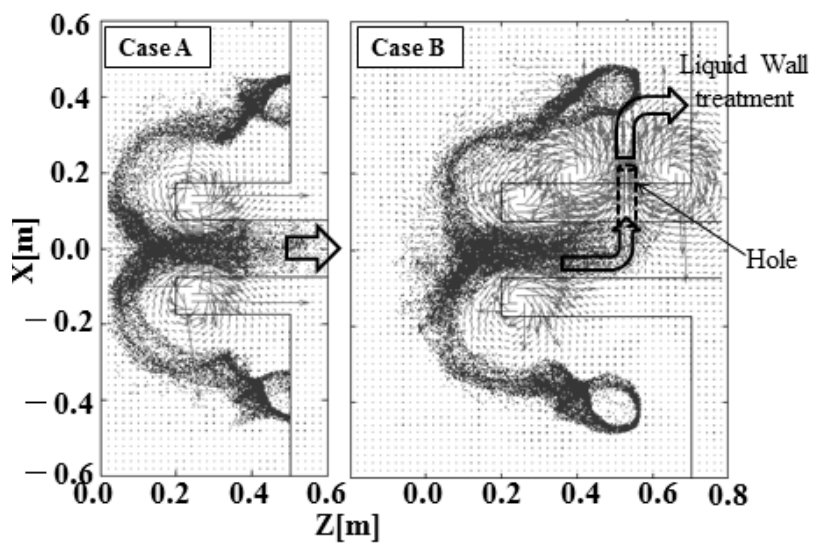

Fig. 3 The vector plot of magnetic field and alpha particle distribution projected onto the $X Z$ plane at $0.53 \mu \mathrm{s}$.

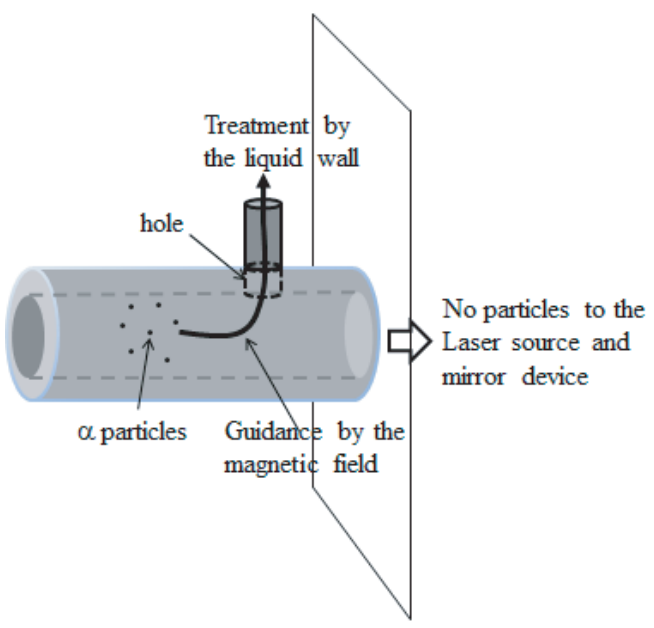

Fig. 4 The illustration of the alpha particles treatment system using the additional coil and the drilled hole at the center of the coil on the beam port.

places (Surface, Inner port, Region A) is shown in Fig. 6. The line "Surface" indicates the ratio between the amounts of alpha particles colliding to the tip surface of the beam port with and without magnetic field. The $100 \%$ of this value is the case without magnetic field. The targeted reduction level of energy of alpha particles colliding to the surface of the beam port is less than $10 \%$ of the load without protection by the magnetic field. The intensity of the magnetic field of $0.9 \mathrm{~T}$ at the center of coil which corresponds to the coil current of $180 \mathrm{kA} \cdot$ turn is enough for achieving the $10 \%$ reduction of the alpha particles. In Case $\mathrm{A}$, this reduction level is almost satisfied. In Case B, this reduction level is also satisfied. The line "Inner port" indicates the ratio between the total amount of alpha particles coming into the inside of the cylindrical beam port with and without magnetic field. The "Inner port" means the amount energy of particles that the particles exist inside of the cylindrical beam port or the particles pass through the inner cylindrical surface as shown the dotted region in 


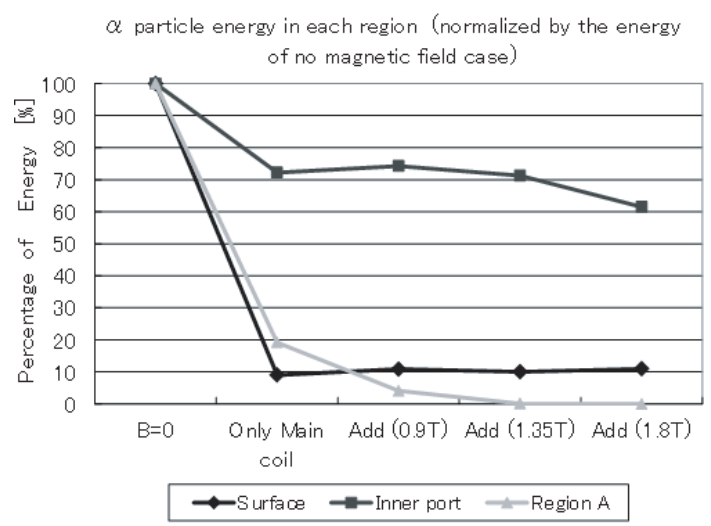

Fig. 5 The percentage of the energy of alpha particles in each region normalized by the each energy in the case without magnetic field.

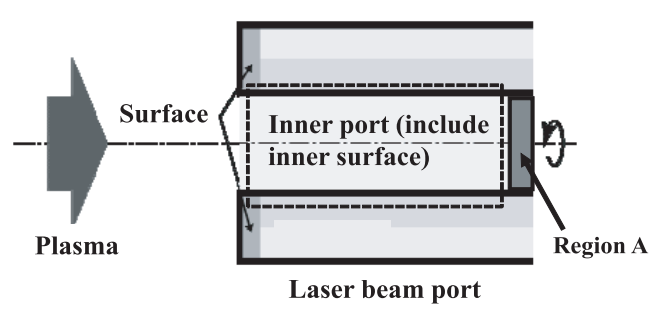

Fig. 6 The definitions of the places.

Fig. 6. While the line "Region A" indicates the ratio between the amount of the alpha particles reaching the "Region A" as shown in Fig. 6 with and without magnetic field. In Case A and B, it is found that the amount of alpha particles coming into the inside of the beam port is $70 \%$ of the energy in the case without magnetic field. A part amount of these particles reaching "Inner port" come to "Region A". In Case A, a lot of alpha particles reach to "Region A", this means the laser source is probably damaged by these particles. In Case B (1.35 T), no alpha particle reaches "Region A". The intensity of $1.35 \mathrm{~T}$ indicates the magnetic flux density at the center of the additional coil. There is the possibility of an appearance of the Rayleigh-Taylor instability for plasma expanding to the magnetic field. But in all cases, this instability was not found since the beta value is lower than unity near the front of the beam port. So, it does not affect the protection of the beam port. In our past research [6], we have conducted the comparison between the experimental results associated with the laserproduced plasma expansion and simulation results using the same hybrid PIC code. The discrepancies between the experimental values and the simulation results in that paper were of the order of $30 \%$. In the present simulations, since the energetic particles produced by the laser are calculated by using the same hybrid PIC code, the situation is quite similar. So the same order of the accuracy can be applied in the present simulations.

\section{Summary}

To evaluate the amount of alpha particles colliding to the surface of the beam port and coming into the inside of the beam port, the numerical simulations by using a $3 \mathrm{D}$ hybrid code have been conducted. As a result, the coil current of $180 \mathrm{kA}$-turn is enough for achieving the $10 \%$ reduction of the alpha particles colliding to the tip surface of the beam port. To prevent these alpha particles reaching to the laser beam source, new configuration of magnetic field generated by the additional hoop coil has been proposed. The intensity of $1.35 \mathrm{~T}$ of the magnetic flux density at the center of the additional coil which is installed at the side of the beam port can guide the alpha particles coming into the inside of the beam port to the outside liquid wall through the drilled hole perfectly. The parallel magnetic field to the tip surface generated by the additional coil prevents the $\mathrm{LiPb}$ liquid on the tip surface of laser beam port from detaching by the Lorenz force between the eddy current in $\mathrm{LiPb}$ and the magnetic field generated by main coil. As the future work, it is necessary for improvement of the protection of the beam port to clarify whether such a large electric current can be sent to the coils and the cooling of these coils can be realized effectively.

\section{Acknowledgement}

This work was performed under the Joint Research of Institute of Laser Engineering, Osaka University.

[1] T. Norimatsu et al., "Conceptual Design of Fast Ignition Power Plant KOYO-F Driven by Cooled YB:YAG Ceramic Laser.", Fusion Science and Technology 52, 893 (2007).

[2] Y. Kozaki et al., "Basic Concepts and a Total Design of Fast Ignition Laser Fusion Reactor”, J. Plasma Fusion Res. 82, 819 (2006).

[3] Y. Kajimura et al., "Numerical study for beam port protection by magnetic fields in the Laser Fusion Reactor KOYOF." Proc. of the IAEA technical meeting on Physics and Technology of IFE targets and chambers, (2007).

[4] Y. Kajimura et al., "Numerical Study for a Protection of Laser Beam Port by Magnetic Fields in the Laser Fusion Reactor KOYO-F.” J. Plasma Fusion Res. 8, 1248 (2009).

[5] D.S. Harned, "Quasineutral Hybrid Simulation of Macroscopic Plasma Phenomena," J. Comp. Phys. 47, 452 (1982).

[6] K.V. Vchivkov et al., "Laser-Produced Plasma Experiments and particle in Cell Simulation to study Thrust Conversion Processes in a Laser Fusion Rocket.”, Jpn. J. Appl. Phys. 42, 6590 (2003). 